\title{
SWITCHING AND PERIODIC CONTROL OF THE BELGIAN CHOCOLATE SYSTEM
}

\author{
Patrizio Colaneri $^{*, 1}$ Didier Henrion ${ }^{* *, * * *, 2}$ \\ * Politecnico di Milano, Piazza Leonardo da Vinci 32, \\ 20133 Milano, Italy \\ ** LAAS-CNRS, 7 avenue du Colonel Roche, \\ 31077 Toulouse, France \\ *** Department of Control Engineering, Faculty of \\ Electrical Engineering, Czech Technical University in \\ Prague, Technická 2, 16627 Prague, Czech Republic
}

\begin{abstract}
The paper aims at providing a switching solution for a parametrized Belgian chocolate stabilization problem posed by Blondel. We show that for any given value of the parameter describing the given transfer function, there exist simple switching and periodic control laws that stabilize the plant. The simplicity in designing these control laws sharply contrasts with the difficulty of designing a non-switching non-periodic control law for this problem. Copyright 2006 IFAC
\end{abstract}

Keywords: Switching, stabilization, periodic systems

\section{INTRODUCTION}

In this paper we consider the linear time-invariant (LTI) system described by the transfer function

$$
G(s)=\frac{s^{2}-1}{s^{2}-2 \delta s+1}
$$

where $\delta$ is a real parameter in the range $[0,1]$. The particular form of this transfer function comes from the $\mathrm{PhD}$ thesis of Vincent Blondel (Blondel, 1994), who offered a kilogram of Belgian chocolate for the solution of each of the following control problems:

Problem 1: Find the range of values for $\delta$ for which there exists an LTI, stable and minimum-phase stabilizing controller for plant (1).

\footnotetext{
1 Partially supported by the Italian National Research Council (CNR), by MIUR project "New methods and algorithms for identification and adaptive control of technological systems"

2 Partially supported by the Grant Agency of the Czech Republic (Grant No. 102/05/0011) and the Ministry of Education of the Czech Republic (Project No. ME 698/2003)
}

Problem 2: Find an LTI, stable and minimumphase stabilizing controller for plant (1) when $\delta=0.9$.

Problem 2 was solved only recently: a controller of eleventh order was found in (Patel et al., 2002) using a randomized search method. Problem 1 is still unsolved. Note however that stabilization is impossible for $\delta=1$ since then an unstable polezero cancellation occurs in plant $G(s)$. According to (Patel et al., 2002) and Prashant Batra ${ }^{3}$, results of complex analysis can be used to prove that there exists a number $\delta^{*}<1$ such that stabilization is possible for all $\delta<\delta^{*}$, but impossible for $\delta \geq \delta^{*}$. In (Burke et al., 2005) a third-order controller was obtained for $\delta=0.9$ but also for the more difficult case $\delta \approx 0.924$, and a fourthorder controller was obtained for $\delta \approx 0.951$, using nonconvex nonsmooth optimization with gradient sampling. Andrey Ghulchak ${ }^{3}$ pointed out then that a third-order controller solving Problem 2

\footnotetext{
3 Personal communication
} 
can be found by a suitable perturbation of necessary stabilizability conditions. Using his coefficient diagram method, Shunji Manabe ${ }^{3}$ has also been able to find a third-order controller solving Problem 2.

One may argue that Blondel's chocolate problems are mainly of academic and mathematical interest. However, in our opinion it is likely that a better understanding of the mathematics of such difficult problems, even though academic ones, can help understand more applied and practical problems. For example, one should recall that control problems with near cancallation of unstable poles and zeros (just as in the Belgian chocolate problem) arise in physically relevant engineering problems, see e.g. the X-29 prototype aircraft design problem or Klein's bicycle design problem mentioned in (Åström, 2000).

The contribution of our paper is to show that simple control laws can be designed for plant (1) and any given value of $\delta<1$, as soon as we relax the assumption that the stable minimumphase controller is LTI. After some preliminaries, in Section 3 we prove the existence of a switching control law that stabilizes the closed-loop system. In Section 4 we show how to construct a first order minimum phase stabilizing periodic controller, parametrized as a function of coefficient $\delta$.

\section{PRELIMINARIES}

First of all, consider a state-space realization for the system with transfer function $G(s)$ :

$$
\begin{gathered}
\dot{x}=A x+B u \\
y=C x+u
\end{gathered}
$$

where

$$
A=\left[\begin{array}{cc}
0 & 1 \\
-1 & 2 \delta
\end{array}\right], B=\left[\begin{array}{l}
0 \\
1
\end{array}\right], C=\left[\begin{array}{ll}
-2 & 2 \delta
\end{array}\right] .
$$

The family of control actions we are looking for is the one defined by

$$
u(t)=F(t) y(t)
$$

where $F(\cdot)$ is a suitable periodic function of period $T$. Notice that $F(t)$ must be different from 1 (the high frequency gain) in order to have a well behaved dynamic closed-loop system. Letting

$$
K(t)=\frac{F(t)}{1-F(t)}
$$

the closed-loop system dynamic matrix becomes

$\hat{A}(t)=A+B K(t) C=\left[\begin{array}{cc}0 & 1 \\ -1-2 K(t) & 2 \delta+2 \delta K(t)\end{array}\right]$.

It is well known that the (closed-loop) periodic system is asymptotically stable if and only if the characteristic multipliers associated with $\hat{A}(\cdot)$ are inside the open unit disk. These characteristic multipliers are the eigenvalues of the monodromy matrix $\Phi(\tau+T, \tau)$, where $\Phi(t, \tau)$ is the transition matrix satisfying

$$
\dot{\Phi}(t, \tau)=\hat{A}(t) \Phi(t, \tau), \quad \Phi(\tau, \tau)=I .
$$

The eigenvalues of $\Phi(\tau+T, \tau)$ do not depend on the tag time $\tau$. Very useful is the celebrated Jacobi formula

$$
\operatorname{det} \Phi(\tau+T, \tau)=\exp \left(\int_{0}^{T} \operatorname{trace}(\hat{A}(s)) d s\right) .
$$

Hence a necessary condition for asymptotic stability is that the trace of the periodic matrix $\hat{A}(\cdot)$ is negative in the average. In our case the necessary condition is given by

$$
T+\int_{0}^{T} K(t) d t<0
$$

This condition is quite easy to be satisfied. However, it is only necessary. Extensive simulations have shown that it is impossible to achieve stability of $\hat{A}(\cdot)$ in (5) with a bounded and smooth periodic gain $K(\cdot)$. Equivalently, we can say that does not exists a smooth periodic function $F(\cdot)$ which is capable to stabilize the closed-loop system without assuming the forbidden value $F(\bar{t})=1$ at some time point $\bar{t}$.

For further information on the theory of periodic systems in polynomial form the reader is referred to (Colaneri, 2005).

\section{SWITCHING CONTROL}

In this section we further particularize the class of the periodic control law. Indeed, we restrict this class to that of piecewise constant laws of the form

$$
K(t)=\left\{\begin{array}{l}
K_{1}, t \in[0, T / 2) \\
K_{2}, t \in[T / 2, T) .
\end{array}\right.
$$

For simplicity we take $T=2$, so that the monodromy matrix results in

$$
\Phi(2,0)=\exp \left(\hat{A}_{2}\right) \exp \left(\hat{A}_{1}\right)
$$

where

$$
\hat{A}_{i}=\left[\begin{array}{cc}
0 & 1 \\
-1-2 K_{i} & 2 \delta+2 \delta K_{i}
\end{array}\right], \quad i=1,2 .
$$

Moreover, the necessary condition simplifies to

$$
K_{1}+K_{2}<-2 .
$$

The eigenvalues of $\Phi(2,0)$, or characteristic multipliers, are complicated functions of $K_{1}$ and $K_{2}$ involving exponentials and radicals, even though an explicit closed-form expression can be obtained 


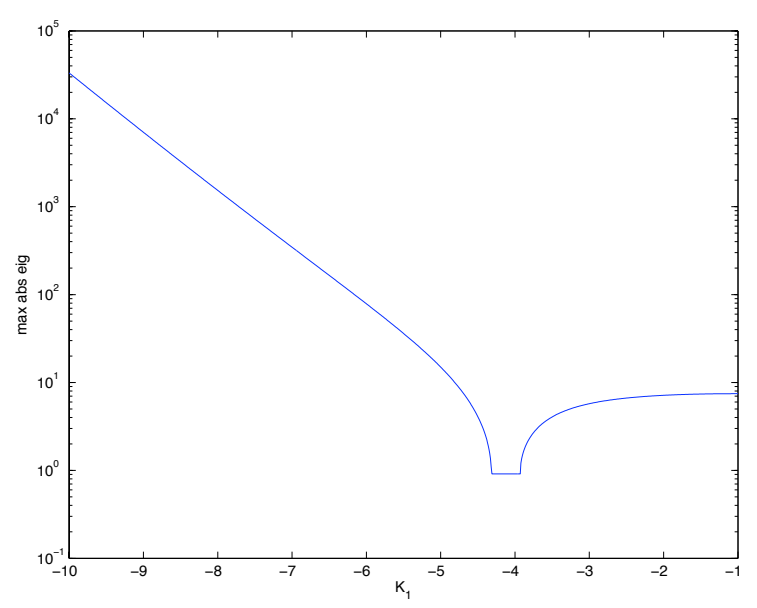

Fig. 1. Maximum modulus of the characteristic multipliers as a function of $K_{1}$ for $\delta=0.9$.

using e.g. Maple. We will not use this expression however. A simpler way to solve the stabilization problem is to fix

$$
K_{1}+K_{2}=-2-\epsilon
$$

where $\epsilon$ is a given strictly positive real number, so that the necessary condition is satisfied. Finally, inverting (4) it follows

$$
F(t)=\frac{K(t)}{1+K(t)}=\left\{\begin{array}{l}
F_{1}, t \in[0, T / 2) \\
F_{2}, t \in[T / 2, T)
\end{array}\right.
$$

where

$$
F_{i}=\frac{K_{i}}{1+K_{i}}, \quad i=1,2 .
$$

For our experiments we chose $\epsilon=0.1$. In Fig. 1, we represent the maximum modulus of the characteristic multipliers as a function of $K_{1}$ for $\delta=0.9$. We can observe the typical nonsmooth (here nonLipschitz) behavior of this function. In Fig. 2 the minimum over $K_{1}$ of this function is represented as a function of $\delta$. Finally, Fig. 3 shows the plot of $F_{1}$ and $F_{2}$ as functions of $\delta$. To this end, we have taken 2000 equidistant values of $\delta$ in the interval $[0.8,0.9999]$, and for each value we have chosen $K_{1}<-1$ that minimizes the maximum modulus of the characteristic multipliers. This explains the nonregularity of the curves. The restriction $K_{1}<-1$ has been enforced to have $F_{1}>1$ for all values of $\delta$.

As apparent, the value of the maximum modulus of the characteristic multipliers is less than one so that asymptotic stability is ensured. As for the dependence of $F_{1}$ and $F_{2}$, we already pointed out that an analytic dependence is hard to be found. The figure shows that stabilization is possible for every $\delta$ in the fixed range.

The curves of $F_{1}$ and $F_{2}$ can be approximated by data-fitting. In Fig. 4, least-squares approximations of $F_{1}(\delta)$ are shown with polynomials of first, third and tenth order. In Fig. 5 the maximum modulus of the characteristic multipliers due to the three polynomials is plotted.

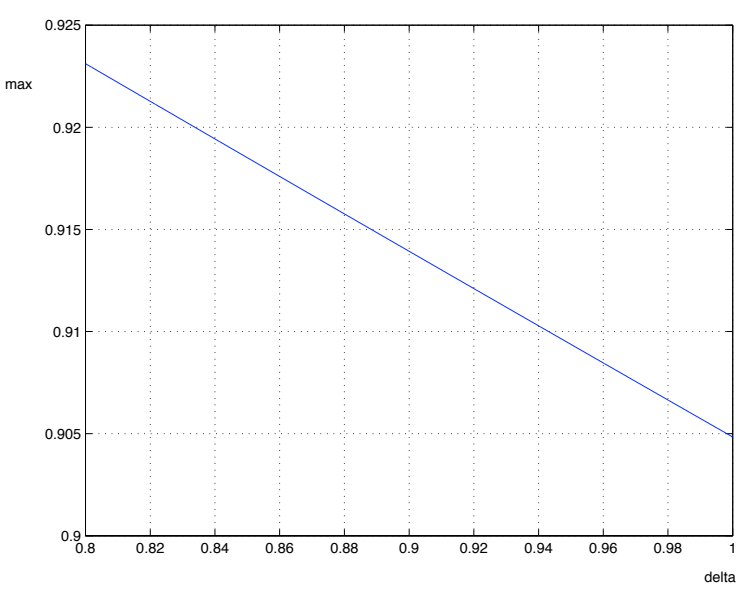

Fig. 2. Maximum modulus of the characteristic multipliers as a function of $\delta$.

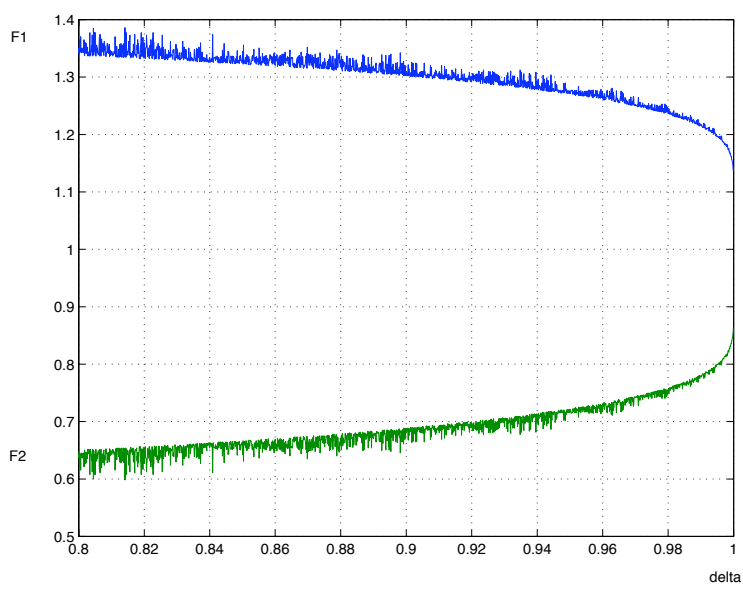

Fig. 3. $F_{1}$ (up) and $F_{2}$ (down) as functions of $\delta$.

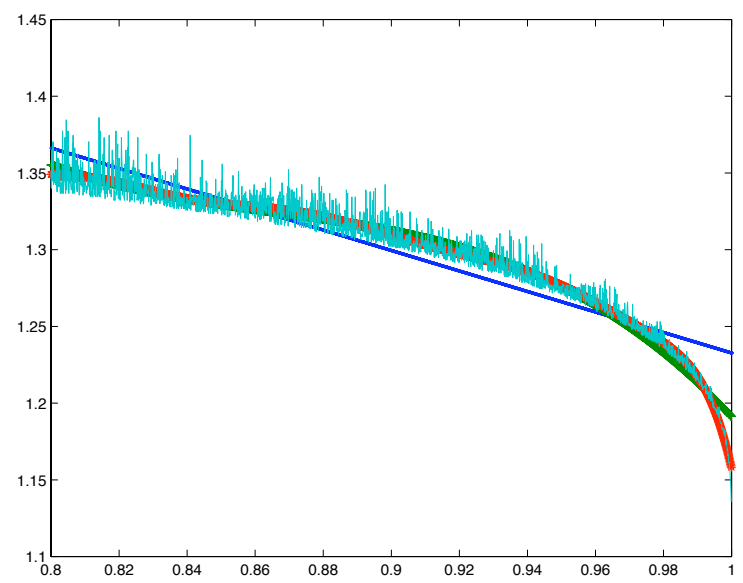

Fig. 4. Polynomial approximations of $F_{1}$ to 1 st order, 3rd order and 10th order.

Easy numerical computations show (see Fig. 5) that the first order polynomial ensures stability up to $\delta=0.8897$, the third order polynomial up to $\delta=0.9716$ and the tenth order polynomial up to $\delta=0.994$. 


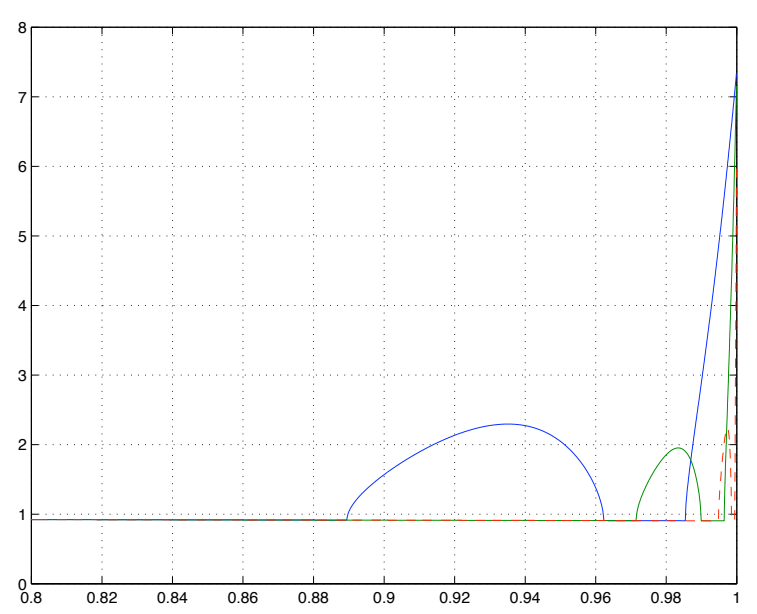

Fig. 5. Maximum modulus of the characteristic multipliers for the three polynomials. First order polynomial (stability till $\delta=0.8897$ ), third order polynomial (stability till $\delta=$ $0.9716)$ ) and tenth order polynomial (stability till $\delta=0.994)$ )

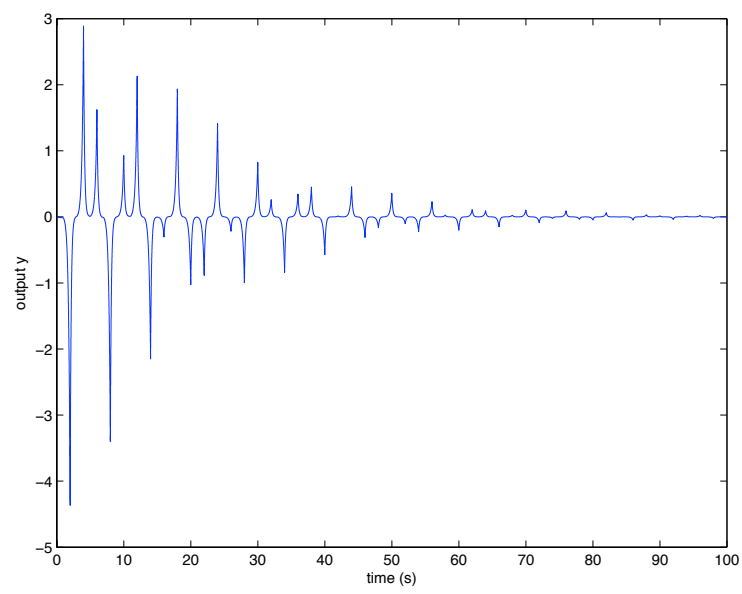

Fig. 6. Output response for $\delta=0.9$ and a switching control law with $K_{1}=-4.2200$ and $K_{2}=2.1200$.

In order to validate the switching control law (7), we implemented it with Simulink, in the case $\delta=0.9$. For this value of $\delta$, we obtained

$$
K_{1}=-4.2200, K_{2}=2.1200 \text {. }
$$

To avoid numerical integration problems due to discontinuous dynamics, we made our simulations on an open-loop plant (1) discretized with a sampling time of $0.001 \mathrm{~s}$ much smaller than the period $T=2 s$ of control law switching. To generate the discrete open-loop model we used the Matlab command c2d with the default method of zeroorder hold on the input. Closed-loop output signal dynamics are represented in Fig. 6 and 7, for a discretized open-loop plant state (arbitrarily) initialized at $(0.1,0.1)$ at $t=0$. We used the default variable-step (automatic maximum step size) discrete solver of Simulink.

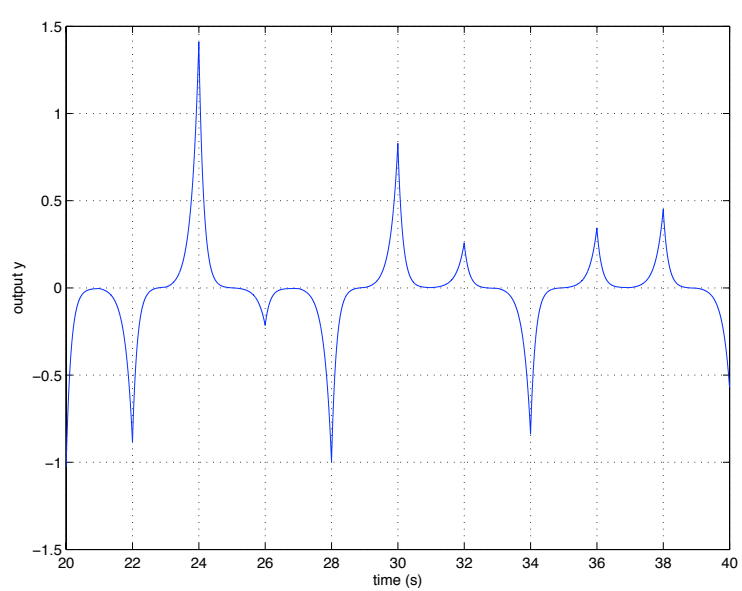

Fig. 7. On this zoom of Fig. 6, we can observe the half-period $T / 2=1 s$ of control law switching.

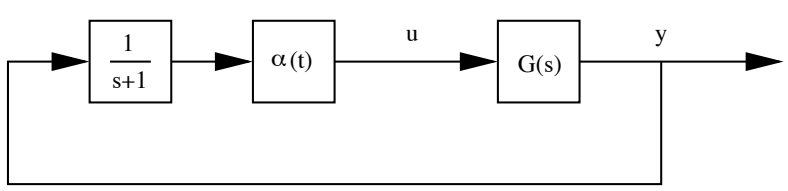

Fig. 8. First order compensator.

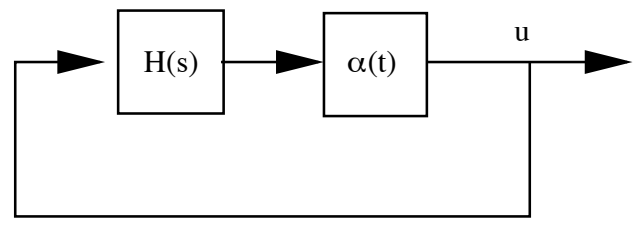

Fig. 9. Equivalent scheme.

Before closing this section, notice again that the values of the stabilizing piecewise constant gain $F(\cdot)$ is such that $1 \in\left[F_{2}, F_{1}\right]$. Hence, a stabilizing smooth function $F(\cdot)$ cannot be built starting from the extreme points $F_{1}$ and $F_{2}$, since it would include the forbidden value $F(\bar{t})=1$ at some time point $\bar{t}$.

\section{FIRST ORDER PERIODIC CONTROLLER}

In this section we try to find a periodic controller of first order that stabilizes the system. The difference with respect to the previous section is that we look for a periodic system with continuous parameters. As already said it does not exist any continuous periodic gain (zero order system) capable of stabilizing the system. Hence we move toward a first order system and consider the scheme depicted in Fig. 8. The equations of the regulator are:

$$
\begin{aligned}
& \dot{\xi}=-\xi+y \\
& u=\alpha(t) \xi
\end{aligned}
$$


Notice that the regulator cancels the stable zero of the system. Hence, concerning stability, the closed-loop system can be given an equivalent structure as shown in Fig. 9, where

$$
H(s)=\frac{s-1}{s^{2}-2 \delta s+1} .
$$

The closed-loop matrix is:

$$
A(t)=\left[\begin{array}{cc}
0 & 1 \\
-1-\alpha(t) & \alpha(t)+2 \delta
\end{array}\right] .
$$

The characteristic polynomial of $A(\cdot)$ is

$$
p(\sigma)=\sigma^{2}-(\alpha(t)+2 \delta) \sigma+1+\alpha(t)
$$

where $\sigma$ stands for the derivative operator. We aim at finding a periodic function $\alpha$ such that the characteristic exponents of $A(\cdot)$ are in the open left half-plane. These characteristic exponents coincide with the average value of the periodic functions $p_{1}$ and $p_{2}$ satisfying the operator equation

$p(\sigma)=\left(\sigma-p_{1}\right)\left(\sigma-p_{2}\right)=\sigma^{2}-\left(p_{1}+p_{2}\right) \sigma+p_{2} p_{1}-\dot{p}_{2}$.

Notice that the derivative of the parameter $p_{2}$ enters the gain, thanks to the non commutative rule $\sigma p_{2}=p_{2} \sigma+\dot{p}_{2}$. Hence, indicating by av $(\cdot)$ the mean value of a periodic function, the stability condition is

$$
\operatorname{av}\left(p_{1}\right)<0, \quad \operatorname{av}\left(p_{2}\right)<0
$$

with

$$
\begin{aligned}
\alpha+2 \delta & =p_{1}+p_{2} \\
\alpha+1 & =p_{2} p_{1}-\dot{p}_{2} .
\end{aligned}
$$

Equivalently, the set of all stabilizing periodic functions $\alpha(t)$ can be written as

$$
\begin{aligned}
& \alpha=\frac{-p_{2}^{2}+2 \delta p_{2}-\dot{p}_{2}}{1-p_{2}} \\
& 0>\operatorname{av}\left(p_{2}\right) \\
& 0>\operatorname{av}\left(\frac{-1+2 \delta-p_{2}-\dot{p}_{2}}{1-p_{2}}\right) .
\end{aligned}
$$

One way to solve the problem is to fix the structure of $p_{2}$ and then optimize the free parameters in order to satisfy (16). For simplicity we took a period equal to $T=1$, and the parameter $p_{2}$ as follows

$$
p_{2}(t)=-0.3-\epsilon(\delta)-1.3 \cos (2 \pi t)
$$

where $\epsilon(\delta)$ is a small number depending on $\delta$. We have chosen $\delta$ ranging from $\delta=0.8$ to $\delta=0.999$. It can be proven by simulation that $\epsilon(\delta)=0.00001$ can be chosen independent of $\delta$ in all the given range. In Fig. 10 the function $\alpha(t)$ given by (14) associated with this constant choice and $\delta=0.999$ is plotted. The closed loop characteristic exponents are

$$
\operatorname{av}\left(p_{2}\right)=-0.3-\epsilon(\delta), \quad \operatorname{av}\left(p_{1}\right) \approx-1 .
$$

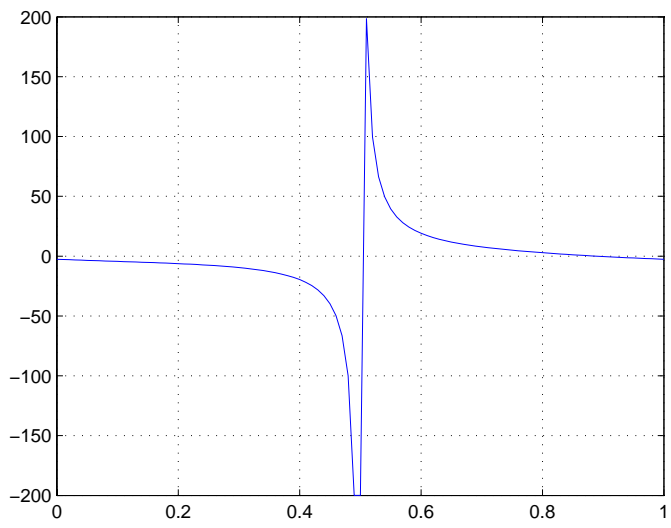

Fig. 10. The gain $\alpha$ for $\delta=0.999$.

Notice that the gain $\alpha(t)$ has a almost piecewise constant form. Indeed, it can be shown that the system can be stabilized by means of a switching function $\alpha(t)$ as well. However, since the overall controller has relative degree equal to one, no restriction on the values assumed by $\alpha(t)$ must be included.

\section{CONCLUDING REMARKS}

In this note we have given some guidelines and rules of thumb to design simple switching and periodic control laws for a benchmark Belgian chocolate stabilization problem. We do not claim that we could solve the problem as stated originally by Blondel, since we relaxed the assumption that the designed stable minimum-phase controller is LTI. However, we believe that the simplicity of the arguments and techniques used to design these control laws could be a good incentive to develop more systematic computer-aided control system design (CACSD) tools for periodic systems.

\section{REFERENCES}

K. J. Åström (2000). Limitations on control system performance. Europ. J. Control, 6(1):2-20.

V. Blondel (1994). Simultaneous stabilization of linear systems. Lecture Notes in Control and Information Sciences, 191, Springer-Verlag, Berlin.

J. V. Burke, D. Henrion, A. S. Lewis, M. L. Overton (2005). Analysis of a Belgian chocolate stabilization problem. $L A A S-C N R S$ Research Report, 05164, Toulouse, France. Submitted for publication.

P. Colaneri (2005). Theoretical aspects of continuos-time periodic systems. Annual Reviews in Control, pp. 206-215.

V. V. Patel, G. Deodhare, T. Viswanath (2002). Some applications of randomized algorithms for control system design. Automatica, 38:2085-2092. 